\title{
Stability patterns for a size-structured population model and its stage-structured
} counterpart

\author{
Zhang, Lai; Pedersen, Michael; Lin, Zhigui
}

Published in:

Mathematical Biosciences

Link to article, DOI:

10.1016/j.mbs.2015.06.014

Publication date:

2015

Document Version

Peer reviewed version

Link back to DTU Orbit

Citation (APA):

Zhang, L., Pedersen, M., \& Lin, Z. (2015). Stability patterns for a size-structured population model and its stagestructured counterpart. Mathematical Biosciences, 267, 109-123. https://doi.org/10.1016/j.mbs.2015.06.014

\section{General rights}

Copyright and moral rights for the publications made accessible in the public portal are retained by the authors and/or other copyright owners and it is a condition of accessing publications that users recognise and abide by the legal requirements associated with these rights.

- Users may download and print one copy of any publication from the public portal for the purpose of private study or research.

- You may not further distribute the material or use it for any profit-making activity or commercial gain

- You may freely distribute the URL identifying the publication in the public portal

If you believe that this document breaches copyright please contact us providing details, and we will remove access to the work immediately and investigate your claim 


\title{
Stability patterns for a size-structured population model and its stage-structured counterpart
}

\author{
Lai Zhang ${ }^{1, \dagger}$, Michael Pedersen ${ }^{2}$, Zhigui $\operatorname{Lin}^{3}$ \\ ${ }^{1}$ Department of Mathematics and Mathematical Statistics, Umeå University, SE-90187, Umeå, Sweden. \\ ${ }^{2}$ Department of Applied Mathematics and Computer Science, Technical University of Denmark, \\ DK-2800, Denmark. \\ ${ }^{3}$ School of Mathematical Science, Yangzhou University, Yangzhou 225002, China. \\ ${ }^{\dagger}$ Corresponding author: lai.zhang@math.umu.se
}

\begin{abstract}
In this paper we compare a general size-structured population model, where a size-structured consumer feeds upon an unstructured resource, to its simplified stage-structured counterpart in terms of equilibrium stability. Stability of the size-structured model is understood in terms of an equivalent delayed system consisting of a renewal equation for the consumer population birth rate and a delayed differential equation for the resource. Results show that the size- and stage-structured models differ considerably with respect to equilibrium stability, although the two models have completely identical equilibrium solutions. First, when adult consumers are superior foragers to juveniles, the size-structured model is more stable than the stage-structured model while the opposite occurs when juveniles are the superior foragers. Second, relatively large juvenile (adult) mortality tends to stabilize (destabilize) the size-structured model but destabilize (stabilize) the stage-structured model. Third, the stability pattern is sensitive to the adultoffspring size ratio in the size-structured model but much less sensitive in the stage-structured model. Finally, unless the adult-offspring size ratio is sufficiently small, the stage-structured model cannot satisfactorily capture the dynamics of the size-structured model. We conclude that caution must be taken when the stage-structured population model is applied, although it can consistently translate individual life history and stage-specific differences to the population level.
\end{abstract}

Keywords: Stability, Size-structured population, Characteristic equation, Life history, Stage-structured population

Mathematics Subject Classification (2010): 92D25, 37N25, 34K20

\section{Introduction}

Ontogenetic development is ubiquitous in nature. During ontogeny, organisms grow from offspring to adult through juvenile stage, resulting in dramatical change in individual physiological states such as body size. As body size governs a majority of individual vital rates such as foraging, consumption, mortality and reproduction (Werner, 1988; Woodward et al., 2005), the change in body size during ontogeny leads to a striking size-dependency of life history. To encompass the size-dependency and track the continuous change in individual physiological states, size-structured population models have been developed (e.g., Metz and Diekmann, 1986; de Roos et al., 1990; Persson et al., 1998; Hartvig et al., 2011). There is a significant body of literature addressing the impacts of size-dependent life history on the dynamics of populations (e.g., de Roos and Persson, 2002; Classen et al., 2002; Zhang et al., 2012) and communities (e.g., Andersen and Beyer, 2006; Datta et al., 2010; Zhang et al., 2013). As an example, a size-structured population model can well capture the single generation cycles in which a single cohort of consumers 
dominates population dynamics throughout its life time and the population composition varies over time between a dominance by juveniles and adults, respectively, (see, de Roos and Persson, 2003).

The significance of size-dependent life history on population dynamics has been well recognised (de Roos et al., 2003) and makes size-structured population models fundamentally different from the unstructured population models. However, this size-dependent life history dramatically increases the complexity of the models and makes analytical tractability intrinsically hard. For this reason, most theoretical studies of size-structured models were investigated by means of numerical simulations (e.g., Claessen and de Roos, 2003). To enhance analytical tractability, de Roos et al. (2008) derived an approximative stagestructured population model from a size-structured population model where a size-structured consumer feeds upon an unstructured resource. The stage-structured counterpart consistently translates assumptions about life history traits such as consumption and growth into a resource-dependent maturation rate. They concluded that these two models have exactly the same predictions under equilibrium conditions, and approximately similar predictions even under non-equilibrium conditions when adult individuals are competitively stronger than juveniles. These two models are the focus of this paper, and we will show analytically that these two models have exactly the same equilibrium solutions.

While cyclic dynamics have been discussed in details in the comparison of the size-structured population model and its stage-structured counterpart (de Roos et al., 2008), global comparison of the stability of these two models has not yet been performed, which impedes the applications of the stage-structured population model. The aim of this paper is to provide such a global analysis of the stability of these two models by analyzing their respective characteristic equations. While stability analysis is straightforward for the stage-structured population model, it is, in stark contrast, mathematically challenging for the size-structured population model.

Modern theory shows that local stability of the size-structured population model can be understood in terms of a delayed system, which is comprised of a renewal equation for the consumer population birth rate and a delayed differential equation for the resource biomass (Diekmann et al., 2010). The mathematical framework for justifying the principle of linearized stability of delay equations is available (e.g., Diekmann et al., 2007, 2010), and one can, based on such a technique, draw rigorous conclusions with respect to the dynamical behavior from an analysis of a characteristic equation. Nevertheless, in spite of the existence of such mathematically rigorous technique, analysis of the characteristic equation is a challenging task as this is typically a rather complicated integral equation. For this reason, de Roos et al. (2010) designed a numerical algorithm to address this issue. However, for the model we will consider, we show that it is possible to derive analytically the characteristic equations, which provides us with an opportunity to assess the global pattern of equilibrium stability.

The paper is organized as follows: In the next section, we describe briefly but completely the sizestructured consumer-resource model. The simplified stage-structured counterpart is presented at the end of this section. In Section 3, we find analytically the equilibria of the size-structured population model, and further provide a rigorous proof that the two considered models have exactly the same equilibria. In Section 4 we investigate the stability of the equilibrium solutions for the size-structured population model by reformulating this model into a delayed system, and we provide an analytical formula for the characteristic equation. In Section 5, we compare the two models with respect to local stability and compute the stability boundary at which the Hopf bifurcation occurs. The paper is finished with a brief conclusion. There are two appendices: Appendix A specifies the real and imaginary part of the characteristic equation of the delayed model and Appendix B shows the stability analysis of the stagestructured population model.

\section{Model description}

In this section we briefly but completely present the aforementioned size-structured consumer-resource model and then the stage-structured counterpart at the end of this section, both of which can be found in de Roos et al. (2008). 
The size-structured population model is described by

$$
\begin{aligned}
\frac{\partial}{\partial t} n(s, t)+\frac{\partial}{\partial t}(g(s, R) n(s, t)) & =-d_{j}(R) n(s, t) \text { for } s_{0} \leq s<s_{m}, \\
g\left(s_{0}, R\right) n\left(s_{0}, t\right) & =\beta\left(s_{m}, R\right) N(t), \\
\frac{d}{d t} N(t) & =g\left(s_{m}, R\right) n\left(s_{m}, t\right)-d_{a}(R) N(t), \\
\frac{d}{d t} R(t) & =G(R)-I \frac{R}{R+H}\left(\int_{s_{0}}^{s_{m}} s n(s, t) d s+q s_{m} N(t)\right),
\end{aligned}
$$

where the first equation describes the dynamics of juvenile individuals $n(s, t)$ (number of individuals per weight), which is a partial differential equation accounting for the ontogenetic growth in body size $s$ (weight). The ontogeny starts from size at birth $s_{0}$ and ceases at maturation size $s_{m}$. The second equation is a boundary condition to accommodate newborn individuals due to the reproduction of adult individuals $N(t)$ (total number of individuals). Adult dynamics is governed by the third equation (2.3). Here $n\left(s_{m}, t\right)=\lim _{s \rightarrow s_{m}^{-}} n(s, t)$. The last equation (2.4) depicts resource dynamics. $G(R)=r R(1-R / K)$ is the resource growth rate in the absence of consumer, and $r$ and $K$ are, respectively, the intrinsic growth rate and the carrying capacity of the resource. Model parameters are derived on the basis of de Roos et al. (2008) and are summarised in Table 1. Below we briefly specify the life history rates of the consumer individuals.

The size-dependent juvenile growth rate and adult birth rate are given as follows

$$
g(s, R)=s w_{j}(R) \quad \text { and } \quad \beta\left(s_{m}, R\right)=\frac{s_{m}}{s_{0}} w_{a}(R),
$$

where

$$
\begin{aligned}
& w_{j}(R)= \begin{cases}\sigma I \frac{R}{H+R}-T, & \text { if } R>\frac{H}{\sigma I / T-1}=R_{j T}, \\
0, & \text { otherwise }\end{cases} \\
& w_{a}(R)= \begin{cases}\sigma q I \frac{R}{H+R}-T, & \text { if } R>\frac{H}{\sigma q I / T-1}=R_{a T}, \\
0, & \text { otherwise }\end{cases}
\end{aligned}
$$

are the net energy balance for juvenile and adult, respectively. $\sigma$ is the assimilation efficiency and $I$ the maximum consumption rate. Here it is assumed that the consumption of the resource follows the Holling type II functional response. $q$ indicates the competitive difference in ability of acquiring resource between juveniles and adults. A value of $q$ below one implies that juveniles are competitively superior to adults whereas higher value than one implies the opposite situation. Additionally, $T$ means the metabolic costs, which is assumed to be size-independent. Clearly when energy fails to meet metabolic costs growth is stopped instantaneously.

Loss of juvenile and adult biomass is due to a constant background mortality and starvation in case that food is insufficient to cover metabolic costs. Specifically,

$$
\begin{aligned}
d_{j}(R) & = \begin{cases}\mu_{j} & \text { if } R \geq R_{j T}, \\
\mu_{j}-v_{j}(R) & \text { otherwise }\end{cases} \\
d_{a}(R) & = \begin{cases}\mu_{a} & \text { if } R \geq R_{a T} \\
\mu_{a}-v_{a}(R) & \text { otherwise }\end{cases}
\end{aligned}
$$

where $\mu_{j}$ and $\mu_{a}$ are constant background mortality on juveniles and adults, and

$$
v_{j}(R)=\sigma I \frac{R}{H+R}-T \quad \text { and } \quad v_{a}(R)=\sigma q I \frac{R}{H+R}-T .
$$


Table 1: Ecological parameters and default values

\begin{tabular}{llll}
\hline Symbol & Value & Unit & Description \\
\hline$s_{0}$ & 0.1 & gram & Size at birth \\
$s_{m}$ & 10 & gram $^{-1}$ & Size at maturation \\
$I$ & 10 & day $^{-1}$ & Maximum ingestion rats per unit biomass \\
$H$ & 1 & gram $/$ a.u. & Half saturation constant for the consumer \\
$T$ & 1 & day $^{-1}$ & Maintenance rate \\
$\sigma$ & 0.5 & - & Efficiency of ingested biomass \\
$q$ & 0.8 & - & Competitive difference in consumption between juveniles and adu \\
$\mu_{j}$ & 0.1 & day & Background mortality on juvenile consumers \\
$\mu_{a}$ & 0.1 & day $^{-1}$ & Background mortality on adult consumers \\
$r$ & 1 & day $^{-1}$ & Resource per capita growth rate \\
$K$ & 2 & gram/a.u. $^{-1}$ & Resource carrying capacity \\
\hline
\end{tabular}

The stage-structured population model, simplified from the size-structured population model (2.1)(2.4), reads as follows

$$
\begin{aligned}
\frac{d J(t)}{d t} & =w_{a}(R) A+v_{j}(R) J-\gamma\left(w_{j}(R)\right) J-\mu_{j} J \\
\frac{d A(t)}{d t} & =\gamma\left(w_{j}(R)\right) J+v_{a}(R) A-w_{a}(R) A-\mu_{a} A \\
\frac{d R(t)}{d t} & =G(R)-I \frac{R}{R+H}(J+q A),
\end{aligned}
$$

where

$$
J(t)=\int_{s_{0}}^{s_{m}} \operatorname{sn}(s, t) d s \text { and } A(t)=s_{m} N(t)
$$

are the total biomass of juvenile and adult consumers, respectively. Moreover,

$$
\gamma(x)=\frac{x-\mu_{j}}{1-\left(s_{0} / s_{m}\right)^{1-\mu_{j} / x}} \quad \text { and } \quad \gamma\left(\mu_{j}\right)=-\frac{\mu_{j}}{\ln \left(s_{0} / s_{m}\right)}
$$

are introduced notations. We refer readers to de Roos et al. (2008) for the details of the simplification.

\section{Steady states}

\subsection{Equilibrium of size-structured population model}

In this section we derive analytically the steady states of system (2.1)-(2.4), and thus starvation does not occur. Thus equilibrium solutions can be solved from the following stationary equations

$$
\begin{aligned}
0 & =w_{j}\left(R^{*}\right) \frac{d\left(s n^{*}(s)\right)}{d s}+\mu_{j} n^{*}(s), \\
0 & =s_{0} w_{j}\left(R^{*}\right) n^{*}\left(s_{0}\right)-\frac{s_{m}}{s_{0}} w_{a}\left(R^{*}\right) N^{*}, \\
0 & =\mu_{a} N^{*}-s_{m} w_{j}\left(R^{*}\right) n^{*}\left(s_{m}\right), \\
0 & =G\left(R^{*}\right)-\frac{I R^{*}}{H+R^{*}}\left(\int_{s_{0}}^{s_{m}} s n^{*}(s) d s+q s_{m} N^{*}\right) .
\end{aligned}
$$


Clearly there is always a trivial equilibrium solution $\left(n^{*}(s), N^{*}, R^{*}\right)=(0,0, K)$, which is, however, out of our interests. In the following, we focus on the positive equilibrium solution $E^{*}=\left(n^{*}(s), N^{*}, R^{*}\right)$. We assume $R_{j T}<R^{*}<K$ to ensure that $w_{j}\left(R^{*}\right)>0$.

Integrating the first equation in (3.1) from $s_{0}$ to $s_{m}$ yields

$$
n^{*}(s)=n^{*}\left(s_{0}\right)\left(\frac{s}{s_{0}}\right)^{-\left(1+\frac{\mu_{j}}{w_{j}\left(R^{*}\right)}\right)} .
$$

Then, substituting equation (3.2) into the third equation in (3.1) gives rise to

$$
N^{*}=\frac{n^{*}\left(s_{0}\right)}{\mu_{a}} s_{m} w_{j}\left(R^{*}\right)\left(\frac{s_{m}}{s_{0}}\right)^{-\left(1+\frac{\mu_{j}}{w_{j}\left(R^{*}\right)}\right)} .
$$

Combining equation (3.3) and the second equation of (3.1), we find

$$
Z_{1}\left(R^{*}\right) \equiv \frac{w_{a}\left(R^{*}\right)}{\mu_{a}}\left(\frac{s_{m}}{s_{0}}\right)^{1-\frac{\mu_{j}}{w_{j}\left(R^{*}\right)}}=1
$$

which gives rise to a unique $R^{*}$ whenever feasible because $Z_{1}(R)-1$ is monotonically increasing with respect to $R$.

Finding the equilibrium solutions of juvenile and adult boils down to find the offspring abundance in equilibrium (i.e., $n^{*}\left(s_{0}\right)$ ), which can actually be computed from the last equation of (3.1). Specifically, it follows from equation (3.2) that the total juvenile biomass at equilibrium is

$$
J^{*}=\int_{s_{0}}^{s_{m}} s n^{*}(s) d s=\frac{n^{*}\left(s_{0}\right) s_{0}^{2}}{1-\frac{\mu_{j}}{w_{j}\left(R^{*}\right)}}\left(\left(\frac{s_{m}}{s_{0}}\right)^{1-\frac{\mu_{j}}{w_{j}\left(R^{*}\right)}}-1\right) .
$$

If $w_{j}\left(R^{*}\right)=\mu_{j}$ we have

$$
J^{*}=\lim _{w_{j}\left(R^{*}\right)->\mu_{j}} \frac{n^{*}\left(s_{0}\right) s_{0}^{2}}{1-\frac{\mu_{j}}{w_{j}\left(R^{*}\right)}}\left(\left(\frac{s_{m}}{s_{0}}\right)^{1-\frac{\mu_{j}}{w_{j}\left(R^{*}\right)}}-1\right)=n^{*}\left(s_{0}\right) s_{0}^{2} \ln \left(\frac{s_{m}}{s_{0}}\right) .
$$

From the second equation of (3.1) and equation (3.4) we see that the total biomass of adult individuals at equilibrium is

$$
A^{*}=s_{m} N^{*}=\frac{n^{*}\left(s_{0}\right) s_{0}^{2} w_{j}\left(R^{*}\right)}{\mu_{a}}\left(\frac{s_{m}}{s_{0}}\right)^{1-\frac{\mu_{j}}{w_{j}\left(R^{*}\right)}} .
$$

From this equation we can find $N^{*}$. Finally it follows from the last equation in (3.1) that

$$
G\left(R^{*}\right)=\frac{I R^{*}}{R^{*}+H}\left\{\frac{n^{*}\left(s_{0}\right) s_{0}^{2}}{1-\frac{\mu_{j}}{w_{j}\left(R^{*}\right)}}\left(\left(\frac{s_{m}}{s_{0}}\right)^{1-\frac{\mu_{j}}{w_{j}\left(R^{*}\right)}}-1\right)+q \frac{n^{*}\left(s_{0}\right) s_{0}^{2} w_{j}\left(R^{*}\right)}{\mu_{a}}\left(\frac{s_{m}}{s_{0}}\right)^{1-\frac{\mu_{j}}{w_{j}\left(R^{*}\right)}}\right\} .
$$

Since $R^{*}$ is determined from equation (3.4), we can find $n^{*}\left(s_{0}\right)$ from equation (3.8). Once $R^{*}$ and $n^{*}\left(s_{0}\right)$ are found, equilibrium juvenile size distribution $\left(n^{*}(s)\right)$ and adult biomass $\left(N^{*}\right)$ are obtained straightforwardly from equation (3.2) and (3.7). Moreover, the interior equilibrium $E^{*}$ is unique when existing, which can be seen from equation (3.4). It follows from equation (3.8) that $n^{*}\left(s_{0}\right)$ is unique. Eventually, there is at most one interior equilibrium to the system (2.1)-(2.4). For the default parameter values in Table 1 , we have $R^{*}=0.3360, J^{*}=0.0475, A^{*}=0.0796$, and $n^{*}\left(s_{0}\right)=0.1848$. The juvinile biomass distribution is illustrated in Fig. 1A. 

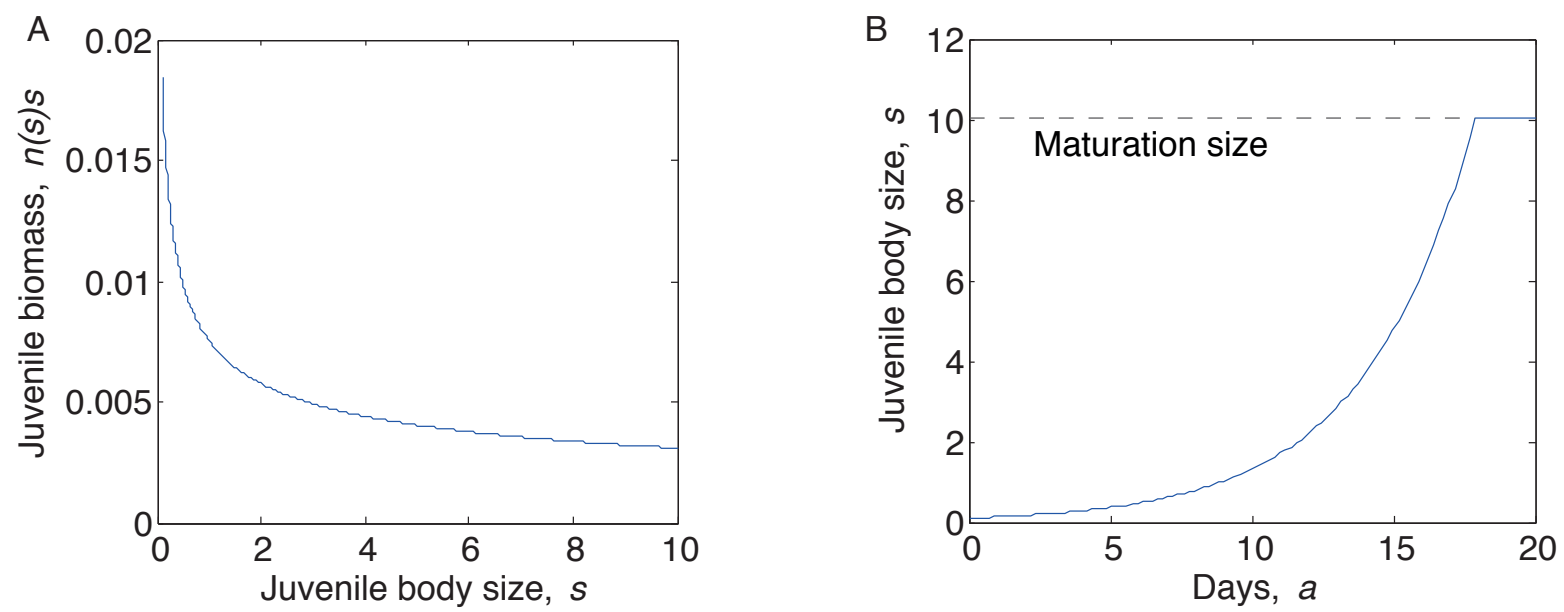

Fig. 1: Juvenile biomass distribution as a function of body size (A) and the growth trajectory of juveniles (B) in equilibrium condition under the default parameter values in Table 1.

Next, we show that $J^{*}, A^{*}$ and $R^{*}$ are also the equilibrium solutions of the system (2.8). We first find the equilibrium solution of the stage-structured population model (2.8), and then prove that the equilibrium solution is exactly the same as that of the size-structured population model. Here we focus on the positive equilibrium solution. Again we assume starvation mortality does not occur under equilibrium conditions, and thus from equation (2.7) we have $v_{j}\left(R^{*}\right)=w_{j}\left(R^{*}\right)$ and $v_{a}\left(R^{*}\right)=w_{a}\left(R^{*}\right)$.

The stationary equations of the stage-structured population model are given by

$$
\begin{aligned}
0 & =w_{a}\left(R^{*}\right) A+w_{j}\left(R^{*}\right) J^{*}-\gamma\left(w_{j}\left(R^{*}\right)\right) J^{*}-\mu_{j} J^{*}, \\
0 & =\gamma\left(w_{j}\left(R^{*}\right)\right) J^{*}-\mu_{a} A^{*} \\
0 & =G\left(R^{*}\right)-\frac{I R^{*}}{R^{*}+H}\left(J^{*}+q A^{*}\right) .
\end{aligned}
$$

Here we use the same notations for the equilibrium solutions as in the size-structured population model because they have exactly the same equilibrium solutions as demonstrated below. From the first two equations of (3.9) we derive that

$$
Z_{2}(R) \equiv \frac{w_{a}(R) \gamma\left(w_{j}(R)\right)}{\mu_{a}\left(\gamma\left(w_{j}(R)\right)-w_{j}(R)+\mu_{j}\right)}=1
$$

If we plug $\gamma\left(w_{j}\left(R^{*}\right)\right)$ into the equation above, it is easy to see that $Z_{2}\left(R^{*}\right)$ is the same as $Z_{1}\left(R^{*}\right)$ in equation (3.4). This means that these two models have the same resource equilibrium if it exists. Once $R^{*}$ is found, the juvenile biomass and adult biomass at equilibrium turn out to be available, that is,

$$
J^{*}=\frac{G\left(R^{*}\right)\left(H+R^{*}\right) /\left(I R^{*}\right)}{1+q \gamma\left(w_{j}\left(R^{*}\right)\right) / \mu_{a}} \quad \text { and } \quad A^{*}=\frac{\gamma\left(w_{j}\left(R^{*}\right)\right)}{\mu_{a}} J^{*} .
$$

Similar formulas for juvenile and adult biomass can also be found in de Roos et al. (2013).

We now show that the juvenile biomass in (3.11) is the same as (3.5). Since $R^{*}$ is the same for both systems, from equation (3.8) we have

$$
\frac{G\left(R^{*}\right)\left(H+R^{*}\right) /\left(I R^{*}\right)}{n^{*}\left(s_{0}\right) s_{0}^{2}}=\frac{1}{1-\frac{\mu_{j}}{w_{j}\left(R^{*}\right)}}\left(\left(\frac{s_{m}}{s_{0}}\right)^{1-\frac{\mu_{j}}{w_{j}\left(R^{*}\right)}}-1\right)+q \frac{w_{j}\left(R^{*}\right)}{\mu_{a}}\left(\frac{s_{m}}{s_{0}}\right)^{1-\frac{\mu_{j}}{w_{j}\left(R^{*}\right)}} .
$$


Let

$$
I=\frac{\frac{1}{1-\frac{\mu_{j}}{w_{j}\left(R^{*}\right)}}\left(\left(\frac{s_{m}}{s_{0}}\right)^{1-\frac{\mu_{j}}{w_{j}\left(R^{*}\right)}}-1\right)+q \frac{w_{j}\left(R^{*}\right)}{\mu_{a}}\left(\frac{s_{m}}{s_{0}}\right)^{1-\frac{\mu_{j}}{w_{j}\left(R^{*}\right)}}}{1+q \gamma\left(w_{j}\left(R^{*}\right)\right) / \mu_{a}} .
$$

If we plug the form of $\gamma\left(w_{j}\left(R^{*}\right)\right)$ (see, equation (2.10)) into the equation above, we have

$$
I=\frac{1}{1-\frac{\mu_{j}}{w_{j}\left(R^{*}\right)}}\left(\left(\frac{s_{m}}{s_{0}}\right)^{1-\frac{\mu_{j}}{w_{j}\left(R^{*}\right)}}-1\right),
$$

which means that the juvenile biomass in equation (3.11) is the same as in equation (3.5). Similarly we can prove that the two systems have the same adult biomass. In conclusion, the two systems have the same positive equilibrium which is unique when existing.

\section{Stability analysis of the size-structured population model}

The aim of this section is to analyze the stability of the positive equilibrium solution (i.e., $\left.\left(n^{*}(s), N^{*}, R^{*}\right)\right)$ of the size-structured population model. However, direct stability analysis is impossible as far as we know because there is no well known mathematical technique by which we can find a stability/instability condition. Nevertheless, the stability can be understood in terms of the solutions of two delayed equations consisting of a renewal equation for the population birth rate of the consumer and a delayed differential equation for the resource biomass (Diekmann et al., 2010). We first present the delayed system and provide an analytic formulation of the equilibrium population birth rate. Then we derive the characteristic equation analytically, which is mathematically challenging and complicated, and thus divided into two subsections.

\subsection{Delayed system}

The delayed system stems from the individual level to the population level by bookkeeping, which introduces a population birth rate of the consumer $b(t)$ and tracks how various individual processes change this population birth rate (Diekmann and Metz, 2010). Specifically, the delayed system reads as follows

$$
\begin{aligned}
b(t)= & \int_{\tau\left(R_{t}\right)}^{\infty} \beta\left(s\left(a, R_{t}\right), R(t)\right) F\left(a, R_{t}\right) b(t-a) d a \\
R^{\prime}(t)= & G(R(t))-\int_{0}^{\tau\left(R_{t}\right)} s\left(a, R_{t}\right) \frac{I R(t)}{H+R(t)} F\left(a, R_{t}\right) b(t-a) d a \\
& -q s_{m} \int_{\tau\left(R_{t}\right)}^{\infty} \frac{I R(t)}{H+R(t)} F\left(a, R_{t}\right) b(t-a) d a,
\end{aligned}
$$

where $R_{t}$ means that

$$
R_{t}(a):=R(t+a), \quad a \in(-\infty, 0],
$$

which is a notion introduced to describe the history of the resource at time $t . s\left(a, R_{t}\right)$ indicates the size of individuals at age $a$, given that it has experienced the history $R_{t}$ in the time interval $[-a, 0]$. $\beta\left(s\left(a, R_{t}\right), R(t)\right)=s_{m} / s_{0} w_{a}(R(t))$ is the adult reproduction rate, independent of the history of the resource. $F\left(a, R_{t}\right)$ is the probability that an individual reaches age $a$ at time $t$ given that it experiences the resource density $R$ in the time interval $[t-a, t] . \tau\left(R_{t}\right)$ is the age at which the consumer individuals mature for a given resource history $R_{t}$, that is, $s\left(\tau\left(R_{t}\right)\right)=s_{m}$. For the detailed explanation of such a delayed system, we refer to Diekmann et al. (2010).

Next we find the equilibrium solutions of the population birth rate $b^{*}$ and resource $R^{*}$. Here again we use the same notation of $R^{*}$ for resource equilibrium as in the size-structured population model, because 
we will show that these two models have exactly the same resource equilibrium. The stationary equations of (4.15) and (4.16) are

$$
\begin{aligned}
b^{*} & =\int_{\tau^{*}}^{\infty} \beta\left(s\left(a, R^{*}\right), R^{*}\right) F\left(a, R^{*}\right) b^{*} d a, \\
G\left(R^{*}\right) & =\int_{0}^{\tau^{*}} s\left(a, R^{*}\right) \frac{I R^{*}}{H+R^{*}} F\left(a, R^{*}\right) b^{*} d a+\int_{\tau^{*}}^{\infty} q s_{m} \frac{I R^{*}}{H+R^{*}} F\left(a, R^{*}\right) b^{*} d a,
\end{aligned}
$$

where $\tau^{*}=\tau\left(R^{*}\right)$ is to be determined. Since we are interested in the positive equilibrium, equation (4.17) is reduced to

$$
1=\int_{\tau^{*}}^{\infty} \beta\left(s\left(a, R^{*}\right), R^{*}\right) F\left(a, R^{*}\right) d a=\frac{s_{m}}{s_{0}} w_{a}\left(R^{*}\right) \int_{\tau^{*}}^{\infty} F\left(a, R^{*}\right) d a,
$$

where we used the fact that $\beta\left(s\left(a, R^{*}\right), R^{*}\right)=s\left(m, R^{*}\right)=s_{m} / s_{0} w_{a}\left(R^{*}\right)$.

Recalling that $F\left(a, R^{*}\right)$ is the survival probability for an individual to reach age $a$ under equilibrium condition, we have

$$
\frac{d F}{d a}= \begin{cases}-\mu_{j} F, & \text { if } \quad 0<a \leq \tau^{*} \\ -\mu_{a} F, & \text { if } \quad a>\tau^{*}\end{cases}
$$

with a initial condition $F(0)=1$. The analytical solution of $F\left(a, R^{*}\right)$ is

$$
F\left(a, R^{*}\right)= \begin{cases}e^{-\mu_{j} a} & \text { if } 0<a \leq \tau^{*} \\ e^{-\mu_{j} \tau^{*}} e^{-\mu_{a}\left(a-\tau^{*}\right)} & \text { if } a>\tau^{*}\end{cases}
$$

Here we use the fact that under equilibrium condition starvation mortality does not occur. Thus mortality of the consumer is independent of the resource biomass. Hence

$$
1=\frac{s_{m}}{s_{0}} w_{a}\left(R^{*}\right) \int_{\tau^{*}}^{\infty} F\left(a, R^{*}\right) d a=\frac{w_{a}\left(R^{*}\right)}{\mu_{a}} \frac{s_{m}}{s_{0}} e^{-\mu_{j} \tau^{*}} .
$$

To determine the maturation age $\tau^{*}$ under equilibrium condition, we consider the growth function

$$
\frac{d}{d a} s\left(a, R^{*}\right)=s\left(a, R^{*}\right) w_{j}\left(R^{*}\right)
$$

for $0 \leq a \leq \tau^{*}$ and $s\left(0, R^{*}\right)=s_{0}$. This equation determines the individual growth trajectory under equilibrium condition. A straightforward calculation yields that

$$
s(a)=s_{0} e^{w_{j}\left(R^{*}\right) a},
$$

and thus

$$
s_{m}=s\left(\tau^{*}\right)=s_{0} e^{w_{j}\left(R^{*}\right) \tau^{*}} .
$$

The maturation age under equilibrium condition is

$$
\tau^{*}=\frac{1}{w_{j}\left(R^{*}\right)} \ln \left(\frac{s_{m}}{s_{0}}\right) .
$$

Plugging equation (4.23) into equation (4.20) yields

$$
1=\frac{w_{a}\left(R^{*}\right)}{\mu_{a}}\left(\frac{s_{m}}{s_{0}}\right)^{1-\frac{\mu_{j}}{w_{j}\left(R^{*}\right)}},
$$


which is exactly the same as equation (3.4), implying that the delayed system and the size-structured population model have exactly the same resource equilibrium as expected. Thus the resource equilibrium $R^{*}$ is obtained by solving equation (4.24).

Now we are ready to find the population birth rate at equilibrium $b^{*}$. In fact,

$$
\begin{aligned}
b^{*} & =\frac{G\left(R^{*}\right)}{\int_{0}^{\tau^{*}} s\left(a, R^{*}\right) \frac{I R^{*}}{H+R^{*}} F\left(a, R^{*}\right) d a+\int_{\tau^{*}}^{\infty} q s_{m} \frac{I R^{*}}{H+R^{*}} F\left(a, R^{*}\right) d a} \\
& =\frac{G\left(R^{*}\right)}{\frac{I R^{*}}{H+R^{*}}\left(\int_{0}^{\tau^{*}} s\left(a, R^{*}\right) F\left(a, R^{*}\right) d a+\frac{q s_{0}}{w_{a}\left(R^{*}\right)}\right)}=\frac{G\left(R^{*}\right)}{\frac{I R^{*}}{H+R^{*}}\left(\frac{s_{0}}{w_{j}(R)-\mu_{j}}\left(\frac{\mu_{a}}{w_{a}\left(R^{*}\right)}-1\right)+\frac{q s_{0}}{w_{a}\left(R^{*}\right)}\right)} .
\end{aligned}
$$

The equilibrium $b^{*}$ is unique due to the uniqueness of $R^{*}$. Under the default parameter values we have the population birth rate $b^{*}=0.0048$, maturation age $\tau^{*}=17.88$ days. The growth trajectory of a juvenile individual under this equilibrium condition is illustrated in Fig. 1B.

\subsection{Characteristic equation}

In this subsection we derive the characteristic equation by which the stability of the equilibrium $b^{*}$ and $R^{*}$ to the delayed system (4.15) and (4.16) can be determined. The characteristic equation is derived by linearizing the delayed system around the positive equilibrium. The linearization of the delayed system is algebraically advanced and mathematically challenging, and we refer readers to Diekmann et al. (2010) for the detailed mathematical derivation. Here we directly present the linearized delayed system after incorporating a small perturbation of the form $b^{*} e^{\lambda t}$ and $R^{*} e^{\lambda t}$ and rearranging the resultant equations:

$$
\begin{gathered}
b^{*}=b^{*} \int_{\tau^{*}}^{\infty} \beta(a) F(a) e^{-\lambda a} d a+\frac{b^{*} R^{*} \beta^{+}}{g^{-}} e^{-\lambda \tau^{*}} K\left(\tau^{*}, \lambda\right) F\left(\tau^{*}\right) \\
+b^{*} R^{*} \int_{\tau^{*}}^{\infty} \beta_{2} F(a) d a+b^{*} R^{*} \int_{\tau^{*}}^{\infty} F(a) \beta_{1}(a) e^{-\lambda a} K(a, \lambda) d a \\
+b^{*} R^{*} e^{-\lambda \tau^{*}} K\left(\tau^{*}, \lambda\right)\left(\frac{g^{+}}{g^{-}}-1\right) \int_{\tau^{*}}^{\infty} \beta_{1}(a) F(a) d a \\
+b^{*} R^{*} \frac{\mu^{-}-\mu^{+}}{g^{-}} K\left(\tau^{*}, \lambda\right) \int_{\tau^{*}}^{\infty} e^{-\lambda a} \beta(a) F(a) d a \\
+b^{*} R^{*} \int_{\tau^{*}}^{\infty} \beta(a) e^{-\lambda a} L(a, \lambda) d a, \\
\lambda R^{*}=G^{\prime}\left(R^{*}\right) R^{*}-b^{*} \int_{0}^{\infty} \nu(a) F(a) e^{-\lambda a} d a-\frac{b^{*} R^{*}\left(\nu^{+}-\nu^{-}\right)}{g^{-}} e^{-\lambda \tau^{*}} K\left(\tau^{*}, \lambda\right) F\left(\tau^{*}\right) \\
-b^{*} R^{*} \int_{0}^{\infty} \nu_{2}(a) F(a) d a-b^{*} R^{*} \int_{0}^{\infty} \nu_{1}(a) e^{-\lambda a} F(a) K(a, \lambda) d a \\
-b^{*} R^{*} e^{-\lambda \tau^{*}} K\left(\tau^{*}, \lambda\right)\left(\frac{g^{+}}{g^{-}}-1\right) \int_{\tau^{*}}^{\infty} \nu_{1}(a) F(a) d a \\
-b^{*} R^{*} \frac{\mu^{-}-\mu^{+}}{g^{-}} K\left(\tau^{*}, \lambda\right) \int_{\tau^{*}}^{\infty} e^{-\lambda a} \nu(a) F(a) d a \\
-b^{*} R^{*} \int_{0}^{\infty} \nu(a) e^{-\lambda a} L(a, \lambda) d a .
\end{gathered}
$$

For the linear system above, the notations are explained below. First, the consumption rate and mortality rate of the consumer individual are, respectively, defined by

$$
\nu\left(a, R^{*}\right):=\left(s\left(a, R^{*}\right) \chi_{\left[0, \tau^{*}\right)}+q s_{m} \chi_{\left[\tau^{*}, \infty\right)}\right) \frac{I R^{*}}{H+R^{*}},
$$


and

$$
\mu=\mu_{j} \chi_{\left[0, \tau^{*}\right)}+\mu_{a} \chi_{\left[\tau^{*}, \infty\right)},
$$

where

$$
\chi_{\left[0, \tau^{*}\right)}(a)= \begin{cases}1, & 0 \leq a<\tau^{*} \\ 0, & a \geq \tau^{*}\end{cases}
$$

Secondly, we introduce short notations for convenience:

$$
\begin{aligned}
& \beta(a):=\beta\left(s\left(a, R^{*}\right), R^{*}\right), \quad \beta_{1}(a):=\left.\frac{\partial}{\partial y} \beta(y, R)\right|_{(y, R)=\left(s\left(a, R^{*}\right), R^{*}\right)}, \\
& \beta_{2}(a):=\left.\frac{\partial}{\partial R} \beta(s, R)\right|_{(s, R)=\left(s\left(a, R^{*}\right), R^{*}\right)}, \quad F(a):=F\left(a, R^{*}\right),
\end{aligned}
$$

and $g_{i}, \nu_{i}$ as well as $\mu_{i}(i=1,2)$ are defined in a similar way. These derivatives will be specified in the next subsection. Thirdly, we define

$$
g^{+}:=\lim _{s \rightarrow>s_{m}^{+}} g\left(s, R^{*}\right) \text { and } g^{-}:=\lim _{s \rightarrow>s_{m}^{-}} g\left(s, R^{*}\right) .
$$

Other symbols such as $\nu^{+}, \nu^{-}, \mu^{-}, \mu^{+}$, and $\beta^{+}$are defined similarly. The discontinuities appearing in the linear system are due to abrupt changes in individual vital rates, which are harmless with respect to integrals.

Finally, the functions $K(a, \lambda)$ and $L(a, \lambda)$ are the solutions of the following two differential equations

$$
\begin{aligned}
\frac{\partial}{\partial a} K(a, \lambda) & =g_{1}(a) K(a, \lambda)+g_{2}(a) e^{\lambda a} \\
\frac{\partial}{\partial a} L(a, \lambda) & =-\mu(a) L(a, \lambda)-\mu_{1}(a) K(a, \lambda) F(a)-\mu_{2}(a) e^{\lambda a} F(a)
\end{aligned}
$$

for $a>0$ and $K(0, \lambda)=L(0, \lambda)=0$. Notice that mortality is stage-dependent, and it is easy to see from (4.30) that $\mu_{1}=\mu_{2}=0$. Thus from equation (4.33) we have $L(a, \lambda) \equiv 0$, which simplifies our linear system (4.26) and (4.27) considerably. We will find $K(a, \lambda)$ analytically in the next subsection.

Equations (4.26) and (4.27) can be rewritten as

$$
\left(\left(\begin{array}{ll}
k_{1} & b^{*} k_{2} \\
k_{3} & G^{\prime}\left(R^{*}\right)+b^{*} k_{4}
\end{array}\right)-\left(\begin{array}{ll}
1 & 0 \\
0 & \lambda
\end{array}\right)\right)\left(\begin{array}{l}
b^{*} \\
R^{*}
\end{array}\right)=0,
$$

where

$$
\begin{aligned}
k_{1}= & \int_{\tau^{*}}^{\infty} \beta(a) F(a) e^{-\lambda a} d a, \\
k_{3}= & -\int_{0}^{\infty} \nu(a) F(a) e^{-\lambda a} d a, \\
k_{2}= & \int_{\tau^{*}}^{\infty} \beta_{2} F(a) d a+\frac{\mu^{-}-\mu^{+}}{g^{-}} K\left(\tau^{*}, \lambda\right) \int_{\tau^{*}}^{\infty} e^{-\lambda a} \beta(a) F(a) d a+\frac{\beta^{+}}{g^{-}} e^{-\lambda \tau^{*}} K\left(\tau^{*}, \lambda\right) F\left(\tau^{*}\right), \\
k_{4}= & -\int_{0}^{\infty} \nu_{2}(a) F(a) d a-\int_{0}^{\infty} \nu_{1}(a) e^{-\lambda a} F(a) K(a, \lambda) d a \\
& -\frac{\mu^{-}-\mu^{+}}{g^{-}} K\left(\tau^{*}, \lambda\right) \int_{\tau^{*}}^{\infty} e^{-\lambda a} \nu(a) F(a) d a-\frac{\left(\nu^{+}-\nu^{-}\right)}{g^{-}} e^{-\lambda \tau^{*}} K\left(\tau^{*}, \lambda\right) F\left(\tau^{*}\right) \\
& -e^{-\lambda \tau^{*}} K\left(\tau^{*}, \lambda\right)\left(\frac{g^{+}}{g^{-}}-1\right) \int_{\tau^{*}}^{\infty} \nu_{1}(a) F(a) d a .
\end{aligned}
$$


The characteristic equation is given by

$$
\operatorname{det}\left(\left(\begin{array}{ll}
k_{1} & b^{*} k_{2} \\
k_{3} & G^{\prime}\left(R^{*}\right)+b^{*} k_{4}
\end{array}\right)-\left(\begin{array}{ll}
1 & 0 \\
0 & \lambda
\end{array}\right)\right)=0
$$

which is equivalent to

$$
\left(k_{1}-1\right)\left(G^{\prime}\left(R^{*}\right)+b^{*} k_{4}-\lambda\right)-b^{*} k_{2} k_{3}=0 .
$$

In principle, one can determine the stability of the delayed system with respect to the equilibrium $b^{*}$ and $R^{*}$ by analysing equation (4.37). However, due to the complexity of $k_{j}(j=1,2,3,4)$, this is almost analytically impossible. In fact, it is sufficient to know when the stability of the equilibrium undergoes a change as a bifurcation parameter increases or decreases. Thus we can proceed by plugging $\lambda=i \omega$ into equation (4.37), and find the critical parameter value at which stability changes. Notice that equation (4.37) is a complex equation. To facilitate our analytical analysis, we rewrite it as two real equations. To this aim, we define real functions $k_{j}^{r}$ and $k_{j}^{i}(j=1,2,3,4)$ such that

$$
k_{j}=k_{j}^{r}+i k_{j}^{i}
$$

where the superinduces $r$ and $i$ refer to "real" and "imaginary". Plugging these functions into equation (4.37), we have an equivalent system of two real equations defined by

$$
H(\omega)=\left(H_{1}(\omega), H_{2}(\omega)\right)^{T}=0,
$$

where the superindex $T$ means the transpose of a matrix and

$$
\begin{aligned}
& H_{1}(\omega)=\left(k_{1}^{r}-1\right)\left(G^{\prime}\left(R^{*}\right)+b^{*} k_{4}^{r}\right)-k_{1}^{i}\left(b^{*} k_{4}^{i}-\omega\right)-b^{*} k_{2}^{r} k_{3}^{r}+b^{*} k_{2}^{i} k_{3}^{i}, \\
& H_{2}(\omega)=\left(k_{1}^{r}-1\right)\left(b^{*} k_{4}^{i}-\omega\right)+k_{1}^{i}\left(G^{\prime}\left(R^{*}\right)+b^{*} k_{4}^{r}\right)-b^{*} k_{2}^{r} k_{3}^{i}-b^{*} k_{2}^{i} k_{3}^{r} .
\end{aligned}
$$

If we now incorporate a parameter $\alpha$ into the notation, we can define a system of two real equations:

$$
H(\alpha, \omega)=0,
$$

the solution of which $\left(\alpha_{c}, \omega_{c}\right)$ is the critical point where stability of the equilibrium solution $\left(b^{*}, R^{*}\right)$ undergoes a qualitative change. The parameter can be any one of $q, K, \mu_{j}$, and $\mu_{a}$, all of which will be considered in the section of numerical results. In the next subsection, we will specify those functions $k_{j}$ $(j=1,2,3,4)$, which makes equations (4.42) solvable.

\subsection{Evaluation of the components of the characteristic equation}

To provide an analytical formula for the characteristic equation we first have to specify the limits (e.g., $\left.g^{-}\right)$defined in (4.31). Since growth is stunted when an individual matures, $g^{+}=0$ and $g^{-}=s_{m} w_{j}\left(R^{*}\right)$. Reproduction occurs only for the adult, and hence $\beta^{+}=\frac{s_{m}}{s_{0}} w_{a}\left(R^{*}\right)$. Finally, it is easy to see that $\nu^{+}=q s_{m} \frac{I R^{*}}{H+R^{*}}$ and $\nu^{-}=s_{m} \frac{I R^{*}}{H+R^{*}}$.

Next we specify the derivatives presented in (4.30). It is easy to see that

$$
\beta_{1}(a)=0, \quad \beta_{2}(a)=\frac{s_{m}}{s_{0}} w_{a}^{\prime}\left(R^{*}\right), \quad \mu_{1}=0, \quad \text { and } \quad \mu_{2}=0,
$$

and

$$
g_{1}(a)=w_{j}\left(R^{*}\right) \quad \text { and } \quad g_{2}(a)=s w_{j}^{\prime}\left(R^{*}\right)
$$

where

$$
w_{j}^{\prime}\left(R^{*}\right)=\sigma I \frac{H}{\left(H+R^{*}\right)^{2}} \quad \text { and } \quad w_{a}^{\prime}\left(R^{*}\right)=\sigma q I \frac{H}{\left(H+R^{*}\right)^{2}} .
$$


In addition, we have

$$
\nu_{1}(a)=\frac{I R^{*}}{H+R^{*}} \chi_{\left[0, \tau^{*}\right)} \quad \text { and } \quad \nu_{2}(a)=\left(s(a) \chi_{\left[0, \tau^{*}\right)}+q s_{m} \chi_{\left[\tau^{*}, \infty\right)}\right) \frac{I H}{\left(H+R^{*}\right)^{2}} .
$$

The analytical solution of $K(a, \lambda)$ in equation (4.32) can be derived from

$$
\frac{\partial}{\partial a} K(a, \lambda)=w_{j}\left(R^{*}\right) K(a, \lambda)+s\left(a, R^{*}\right) w_{j}^{\prime}\left(R^{*}\right) e^{\lambda a}
$$

for $0 \leq a<\tau^{*}$. Straightforward calculation yields that

$$
K(a, \lambda)=e^{w_{j}\left(R^{*}\right) a} \int_{0}^{a} s\left(t, R^{*}\right) w_{j}^{\prime}\left(R^{*}\right) e^{\lambda t} d t=e^{w_{j}\left(R^{*}\right) a} \frac{s_{0} w_{j}^{\prime}\left(R^{*}\right)}{w_{j}\left(R^{*}\right)+\lambda}\left(e^{\left(w_{j}\left(R^{*}\right)+\lambda\right) a}-1\right) .
$$

To obtain an analytical form of the $k_{j}$ in equations (4.35), we have to evaluate the integrals appearing in those functions by assuming that $\lambda$ is purely imaginary. It is easy to see that

$$
k_{1}=\int_{\tau^{*}}^{\infty} \beta(a) F(a) e^{-\lambda a} d a=\frac{\mu_{a}}{\mu_{a}+\lambda} e^{-\lambda \tau^{*}}
$$

and

$$
\begin{aligned}
-k_{3} & =\int_{0}^{\infty} \nu(a) F(a) e^{-\lambda a} d a \\
& =\frac{s_{0} I R^{*}}{H+R^{*}} \int_{0}^{\tau^{*}} e^{\left(w_{j}\left(R^{*}\right)-\mu_{j}-\lambda\right) a} d a+q s_{m} \frac{I R^{*}}{H+R^{*}} \int_{\tau^{*}}^{\infty} F(a) e^{-\lambda a} d a \\
& =\frac{s_{0} I R^{*}}{H+R^{*}} \frac{1}{w_{j}\left(R^{*}\right)-\mu_{j}-\lambda}\left(\frac{\mu_{a}}{w_{a}\left(R^{*}\right)} e^{-\lambda \tau^{*}}-1\right)+q s_{0} \frac{I R^{*}}{H+R^{*}} \frac{1}{w_{a}\left(R^{*}\right)} \frac{\mu_{a}}{\mu_{a}+\lambda} e^{-\lambda \tau^{*}} .
\end{aligned}
$$

In addition, because the adults do not grow and hence $\nu_{1} \equiv 0$, we have

$$
\int_{\tau^{*}}^{\infty} \nu_{1}(a) F(a) d a=0
$$

It remains to evaluate three integrals in order to obtain $k_{2}$ and $k_{4}$, that is,

$$
\begin{aligned}
\int_{\tau^{*}}^{\infty} \beta_{2}(a) F(a) d a= & \frac{s_{m}}{s_{0}} w_{a}^{\prime}\left(R^{*}\right) \int_{\tau^{*}}^{\infty} F(a) d a=\frac{w_{a}^{\prime}\left(R^{*}\right)}{w_{a}\left(R^{*}\right)} \\
\int_{0}^{\infty} \nu_{2}(a) F(a) d a= & \frac{I H}{\left(H+R^{*}\right)^{2}}\left(\int_{0}^{\tau^{*}} s\left(a, R^{*}\right) F(a) d a+\int_{\tau^{*}}^{\infty} q s_{m} F(a) d a\right) \\
= & \frac{I H}{\left(H+R^{*}\right)^{2}}\left[\frac{s_{0}}{w_{j}\left(R^{*}\right)-\mu_{j}}\left(\frac{\mu_{a}}{w_{a}\left(R^{*}\right)}-1\right)+\frac{q s_{0}}{w_{a}\left(R^{*}\right)}\right] \\
\int_{0}^{\infty} \nu_{1}(a) e^{-\lambda a} F(a) K(a, \lambda) d a= & \frac{I R^{*}}{H+R^{*}} \int_{0}^{\tau^{*}} e^{-\lambda a} F(a) e^{w_{j}\left(R^{*}\right) a} \frac{s_{0} w_{j}^{\prime}\left(R^{*}\right)}{w_{j}\left(R^{*}\right)+\lambda}\left(e^{\left(w_{j}\left(R^{*}\right)+\lambda\right) a}-1\right) d a \\
= & \frac{I R^{*}}{H+R^{*}} \frac{s_{0} w_{j}^{\prime}\left(R^{*}\right)}{w_{j}\left(R^{*}\right)+\lambda} \int_{0}^{\tau^{*}} F(a)\left[e^{2 w_{j}\left(R^{*}\right) a}-e^{\left(w_{j}\left(R^{*}\right)-\lambda\right) a}\right] d a \\
& =\frac{I R^{*}}{H+R^{*}} \frac{s_{0} w_{j}^{\prime}\left(R^{*}\right)}{w_{j}\left(R^{*}\right)+\lambda}\left\{\frac{1}{2 w_{j}\left(R^{*}\right)-\mu_{j}}\left(e^{\left(2 w_{j}\left(R^{*}\right)-\mu_{j}\right) \tau^{*}}-1\right)\right. \\
& \left.-\frac{1}{w_{j}(R)-\mu_{j}-\lambda}\left(e^{\left(w_{j}\left(R^{*}\right)-\mu_{j}-\lambda\right) \tau^{*}}-1\right)\right\} \\
& \frac{I R^{*}}{H+R^{*}} \frac{s_{0} w_{j}^{\prime}\left(R^{*}\right)}{w_{j}\left(R^{*}\right)+\lambda}\left\{\frac{1}{2 w_{j}\left(R^{*}\right)-\mu_{j}}\left(\frac{s_{m} \mu_{a}}{s_{0} w_{a}\left(R^{*}\right)}-1\right)\right. \\
& \left.\frac{1}{w_{j}(R)-\mu_{j}-\lambda}\left(\frac{\mu_{a}}{w_{a}\left(R^{*}\right)} e^{-\lambda \tau^{*}}-1\right)\right\} .
\end{aligned}
$$


Finally, we have the analytical forms of $k_{2}$ and $k_{4}$

$$
k_{2}=\frac{w_{a}^{\prime}\left(R^{*}\right)}{w_{a}\left(R^{*}\right)}+\frac{\mu_{j}-\mu_{a}}{s_{m} w_{j}\left(R^{*}\right)} K\left(\tau^{*}, \lambda\right) \frac{\mu_{a}}{\mu_{a}+\lambda} e^{-\lambda \tau^{*}}+\frac{w_{a}\left(R^{*}\right)}{s_{0} w_{j}\left(R^{*}\right)} F\left(\tau^{*}\right) e^{-\lambda \tau^{*}} K\left(\tau^{*}, \lambda\right),
$$

and

$$
\begin{aligned}
-k_{4}= & \frac{I H}{\left(H+R^{*}\right)^{2}}\left[\frac{s_{0}}{w_{j}\left(R^{*}\right)-\mu_{j}}\left(\frac{\mu_{a}}{w_{a}\left(R^{*}\right)}-1\right)+\frac{q s_{0}}{w_{a}\left(R^{*}\right)}\right] \\
& +\frac{I R^{*}}{H+R^{*}} \frac{s_{0} w_{j}^{\prime}\left(R^{*}\right)}{w_{j}\left(R^{*}\right)+\lambda}\left[\frac{1}{2 w_{j}\left(R^{*}\right)-\mu_{j}}\left(\frac{s_{m} \mu_{a}}{s_{0} w_{a}\left(R^{*}\right)}-1\right)-\frac{1}{w_{j}(R)-\mu_{j}-\lambda}\left(\frac{\mu_{a}}{w_{a}\left(R^{*}\right)} e^{-\lambda \tau^{*}}-1\right)\right] \\
& +\frac{\mu_{j}-\mu_{a}}{s_{m} w_{j}\left(R^{*}\right)} \frac{I R^{*}}{H+R^{*}} \frac{q s_{0}}{w_{a}\left(R^{*}\right)} K\left(\tau^{*}, \lambda\right) \frac{\mu_{a}}{\mu_{a}+\lambda} e^{-\lambda \tau^{*}} \\
& +\frac{(1-q)}{s_{m} w_{j}\left(R^{*}\right)} \frac{I R^{*}}{H+R^{*}} e^{-\lambda \tau^{*}} K\left(\tau^{*}, \lambda\right) F\left(\tau^{*}\right)
\end{aligned}
$$

where

$$
K\left(\tau^{*}, \lambda\right)=\lim _{a->\tau^{*}} K(a, \lambda)=e^{w_{j}\left(R^{*}\right) \tau^{*}} \frac{s_{0} w_{j}^{\prime}\left(R^{*}\right)}{w_{j}\left(R^{*}\right)+\lambda}\left(e^{\left(w_{j}\left(R^{*}\right)+\lambda\right) \tau^{*}}-1\right)=\frac{s_{m} w_{j}^{\prime}\left(R^{*}\right)}{w_{j}\left(R^{*}\right)+\lambda}\left(\frac{s_{m}}{s_{0}} e^{\lambda \tau^{*}}-1\right),
$$

and

$$
F\left(\tau^{*}\right)=e^{-\mu_{j} \tau^{*}}=\frac{\mu_{a}}{w_{a}\left(R^{*}\right)} \frac{s_{0}}{s_{m}} .
$$

The real and imaginary parts of $k_{j}(j=1,2,3,4)$ are specified in appendix B.

\section{Numerical results}

In this section we compare the size-structured population model and its stage-structured counterpart in terms of equilibrium stability by analyzing their respective characteristic equations. To begin with, we show how to obtain the critical value of a bifurcation parameter from the characteristic equation (4.42) for the size-structured population model. We consider the default parameter values except for the adult competitive ability $q$ and the resource carrying capacity $K$. We take $q=2$ and let $K$ be a free parameter in equation (4.42). Figure 2 shows the null lines of the equation (4.42). The intersection of these null lines gives rise to the critical point $K_{c}$ at which a Hopf bifurcation occurs. For the considered parameter values, we have $K_{c}=1.25$ and $\omega_{c}=0.85$.

The stability pattern with respect to the parameters of resource carrying capacity $(K)$ and adult competitive ability $(q)$ is presented in Fig. 3. Clearly, the stability patterns are considerably different between the size- and stage-structured models, although both models exhibit instability for high resource carrying capacity. First, for lower resource carrying capacity, the size-structured population model is in general stable with high adult competitive ability, while the stage-structured population model tends to be unstable with increasing adult competitive ability. Second, the stability boundary for the size-structured model is much more complicated than for the stage-structured model. This difference implies higher nonlinear effects of the two bifurcation parameters on the equilibrium stability in the former model than in the latter.

The stability pattern with respect to the parameters of juvenile and adult mortality is presented in Fig. 4. Again, the two models differ considerably in terms of the stability region. There are two interesting observations. First, for relatively low juvenile mortality, high adult mortality stabilizes the stage structured model but destabilizes the size-structured model. Second, for low adult mortality, relatively high juvenile mortality stabilizes the size-structured model but destabilizes the stage-structured model. 


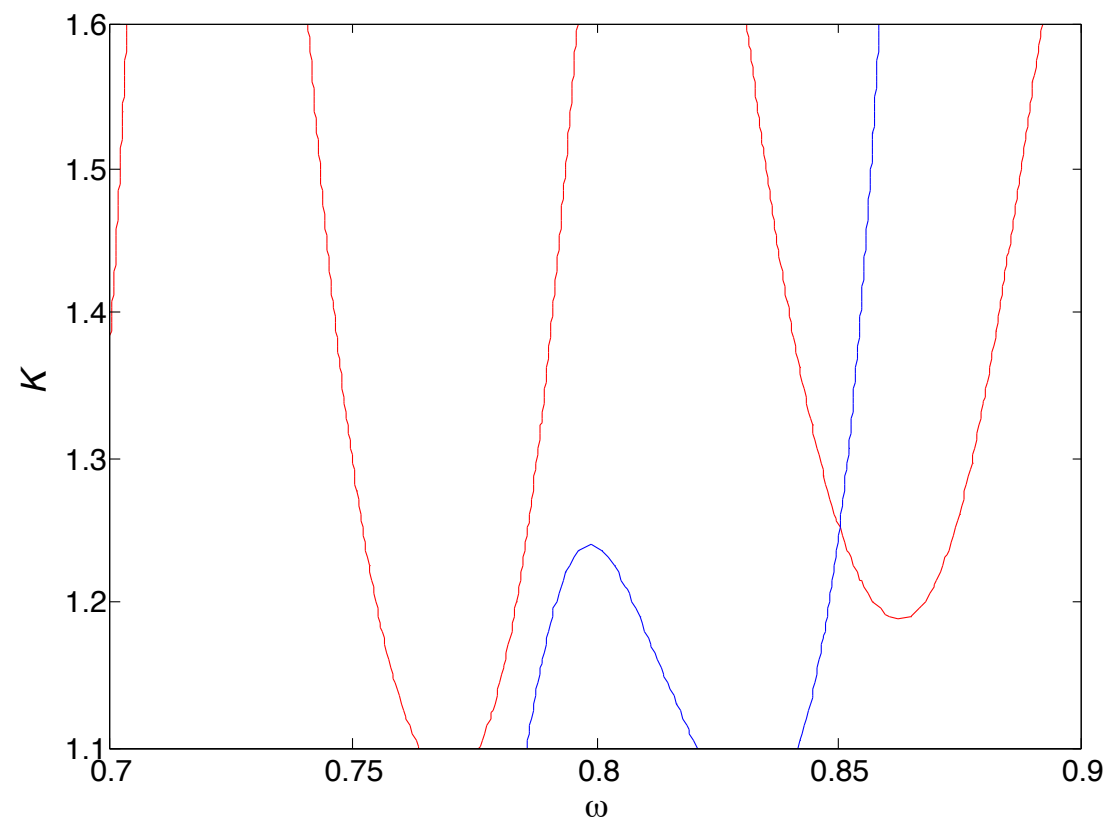

Fig. 2: The null lines of equations (4.42). The intersection point shows the critical value of the considered bifurcation parameter, that is, resource carrying capacity $K$.

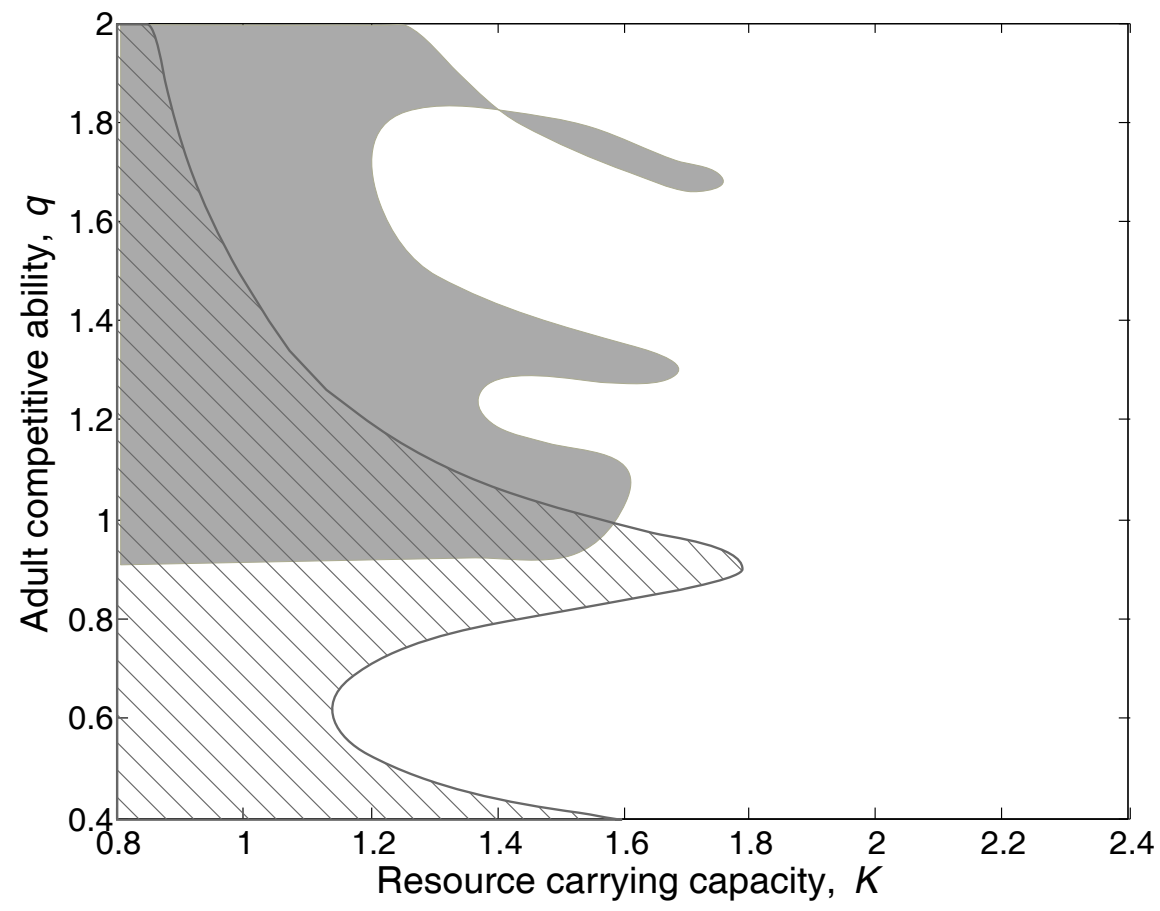

Fig. 3: Stability region of the size-structured population model (shaded area) and the stage-structured population model (hatched area) in the parameter space spanned by the two bifurcation parameters of resource carrying capacity $(K)$ and adult competitive ability $(q)$. Other parameter values are taken as the default values in Table 1 . 


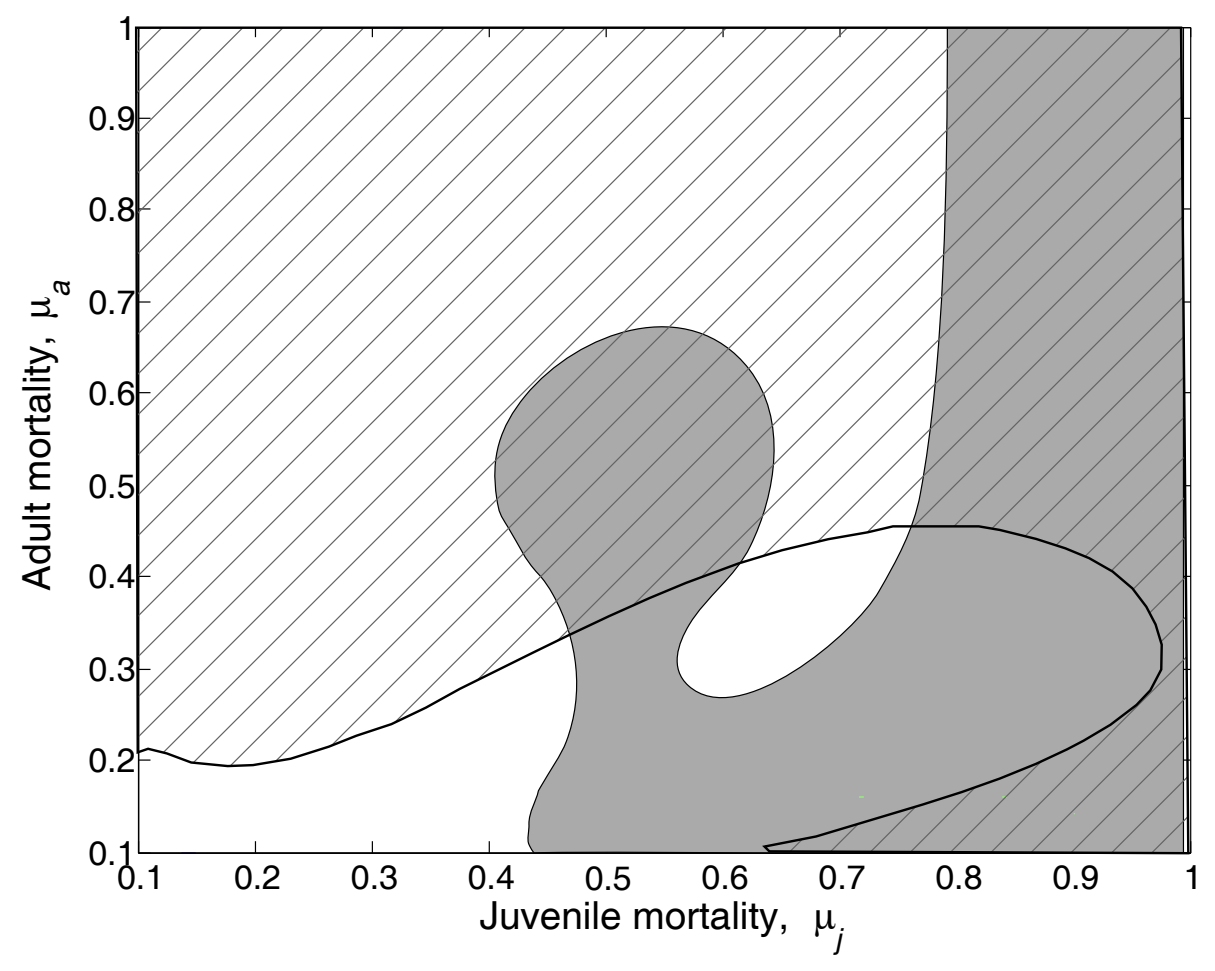

Fig. 4: Stability region of the size-structured population model (shaded area) and the stage-structured population model (hatched area) in the parameter space spanned by the two bifurcation parameters of juvenile $\left(\mu_{j}\right)$ and adult $\left(\mu_{a}\right)$ mortality. Other parameter values are taken as the default values in Table 1.

These findings imply that the size-structured population model is more sensitive to juvenile mortality while the stage-structured population model is sensitive to adult mortality.

Within the instability region, three different cyclic dynamics are predicted from the size-structured population model: the prey escape cycle, single generation cycle, and chaotic cycle while the stagestructured population model only captures the prey escape cycle (Fig. 5). This observation has been reported in details in de Roos et al. (2008). For completeness, we describe briefly these cycles. The prey escape cycle is the traditional predator-prey cycle, where the cycle depends on the characteristics of both prey and predator (de Roos et al., 1990). As seen from Fig. 5A, the cycles of juvenile and adult biomass are highly correlated and in-phase. Its appearance is generally due to the logistic growth and the Holling type II functional response. The single generation cycle depends only on the characteristic of the predator. It shows in Fig. 5B that a newborn cohort of individuals dominates the population dynamics throughout its lifetime and leads to the next dominant cohort. An apparent feature is that the dominance of juvenile and adult biomass alternates as time proceeds. The chaotic cycle results from combination of the former two cycles.

Finally, we investigate the impact of adult-offspring size ratio to the stability of the size- and stagestructured population models in the parameter space spanned by juvenile and adult mortality. Figure 6 reveals that with an increase in the size ratio, the stability pattern is very sensitive to this size ratio in the size-structured population model, but less sensitive in the stage-structured population model. Moreover, it is easy to see that unless the adult-offspring size ratio is sufficiently small (e.g., $s_{m} / s_{0}=2$ ), the stability pattern predicted by these two models are dramatically distinctive. 

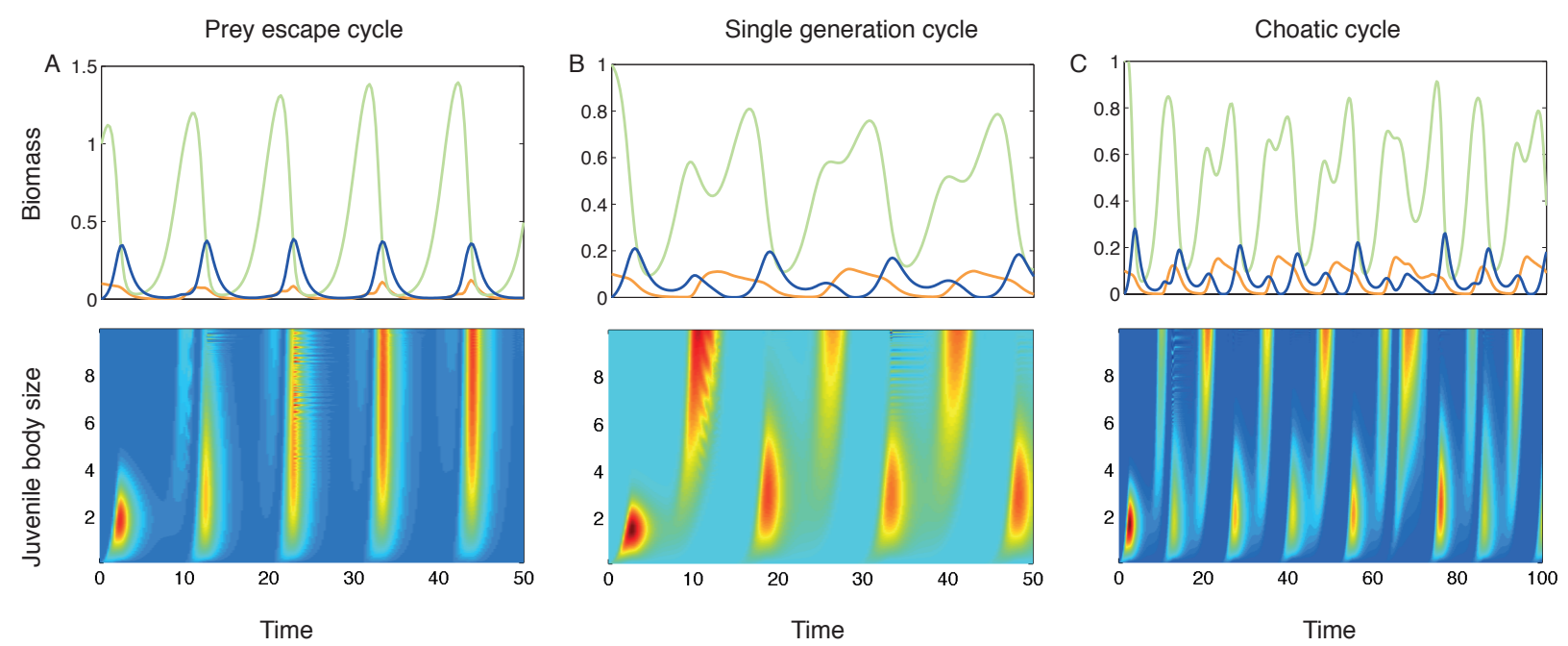

Fig. 5: Three different cycles are captured by the size-structured population model: Prey escape cycle (A), Single generation cycle (B), and Chaotic cycle (C). The top panels show the biomass of the resource (green), total juvenile biomass (blue) and adult biomass (orange). The bottom panels show that biomass of differently sized juvenile individuals, where juvenile biomass is scaled with the equilibrium biomass. The brighter the region, the higher the biomass relative to equilibrium biomass. Parameter values are $q=0.5$ with $K=2(\mathrm{~A}), K=1.25(\mathrm{~B})$, and $K=1.6(\mathrm{C})$. Unvaried parameters are taken as the default values in Table 1.
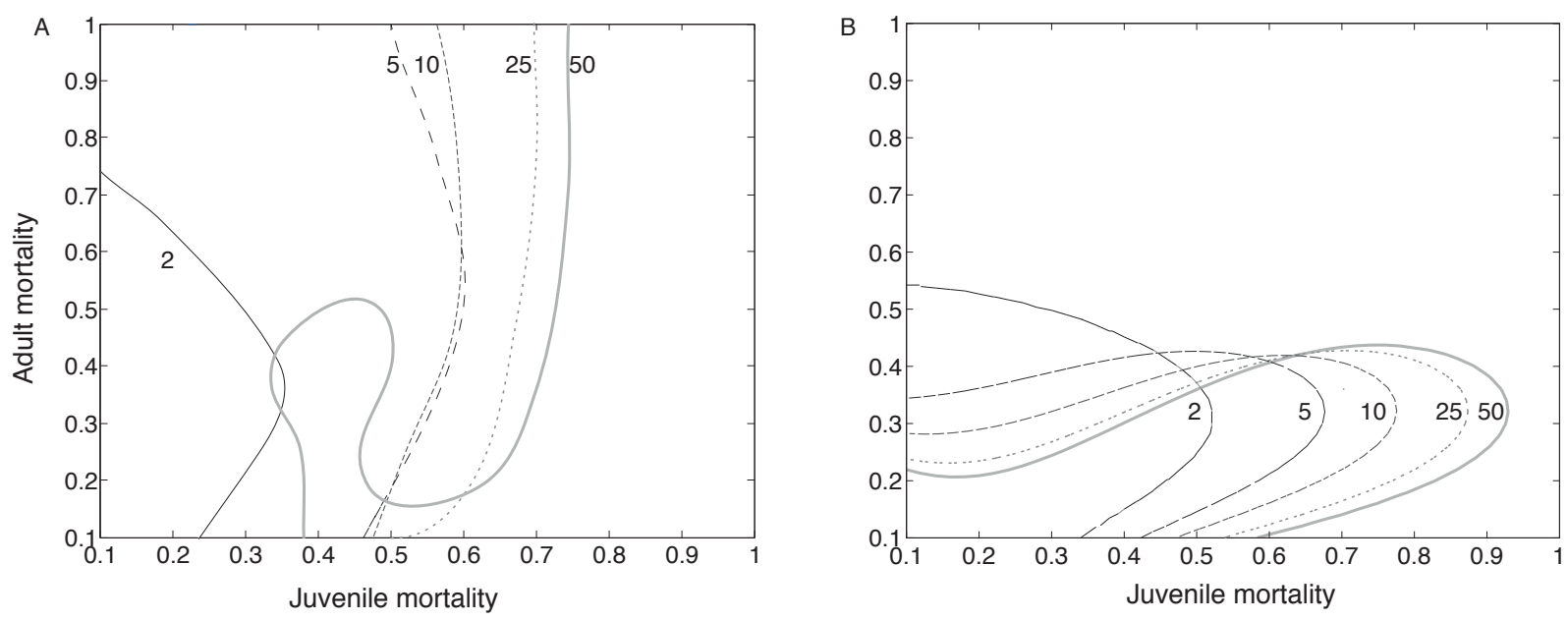

Fig. 6: The stability boundaries of the size-structured population model (A) and the stage-structured population model (B) for different adult-offspring size ratio in the parameter space spanned by juvenile and adult mortality. The numbers indicate the size ratio between adult and offspring, that is, $s_{m} / s_{0}$. 


\section{Conclusions}

In this paper we have compared the size-structured population model and its stage-structured counterpart in terms of the stability of the positive equilibrium. We rigorously proved that these two models have exactly the same positive equilibrium which is actually unique. Stability analysis of the positive equilibrium is straightforward for the stage-structured population model, but mathematically challenging for the size-structured population model. Nevertheless, local stability of the size-structured population model might be seen from its delayed system (Diekmann et al., 2010). The delayed system is comprised of a renewal equation for the consumer population birth rate and a delayed differential equation to account for the resource dynamics. By analyzing the stability of this delayed system, we are able to obtain the stability behavior of the size-structured population model. The characteristic equation of the delayed system was derived analytically, which was used to determine the stability of equilibrium solution.

By comparing the stability patterns in the investigated parameter spaces, we found that although the size-structured model and the stage-structured model lead to completely identical predictions under equilibrium conditions, they differ considerably in terms of equilibrium stability. Specifically, four interesting findings have been discussed. First, for the coexistence of consumer and resource species with low resource carrying capacity, strong adult competitive ability (i.e., $q>1$ ) promotes stability of the size-structured model but tends to destabilize the stage-structured model, while the opposite happens for the two models when juvenile individuals are competitively stronger than adult individuals (Fig. 3). Second, the stage-structured model tends to overestimate the stabilizing effect of the adult mortality but underestimate the stabilizing effect of juvenile mortality (Fig. 4). Third, the stage-structured model is less sensitive to the adult-offspring size ratio compared to the size-structured model (Fig. 6). Finally, small differences between adult and offspring size can significantly differentiate the size-structured model from the stage-structured model (Fig. 6). Apart from the above differences we also showed that the stagestructured population model fails to capture the single generation cycle and the chaotic cycle (Fig. 5). For the detailed discussions of the cyclic dynamics we refer readers to de Roos et al. (2008). We also considered other values for the parameters presented in Table 1, but found that those above discrepancies between the size-structured population model and its stage-structured counterpart are fairly robust. For instance we consider a figure similar to figure 4 but with $q=2$, meaning that adult individuals are competitively stronger than juvenile individuals (Fig. 7), which also shows a dramatic difference between considered two models in terms of equilibrium stability.

The reason for these observed discrepancies is because of the key assumption that the distribution of juveniles is fixed to its equilibrium distribution when simplifying the size-structured population model into a stage-structured population model. The fixation leads to a distributed time delay between the birth and maturation of an individual organism, while in the size-structured population model maturation is a discrete event in individual life history. Thus, in the stage-structured population model maturation from juveniles to adults is described at the population level (i.e., $\gamma\left(w_{j}(w), R\right)$ in system $(2.8)$ ) while at the individual level in the size-structured population model. We conclude that aforementioned key assumption can fundamentally alter the nature of the positive equilibrium, although the simplified stage-structured counterpart can consistently translate assumptions about consumption, growth and bioenergetics of individual consumers into a resource-dependent, population-level maturation rate.

\section{Acknowledgements}

Lai Zhang gratefully acknowledges the financial support from the Swedish Strategic Research Programme eSSENCE.

\section{References}

Andersen KH, Beyer JE (2006) Asymptotic size determines species abundance in the marine size spectrum. Am Nat 168:54-61. 

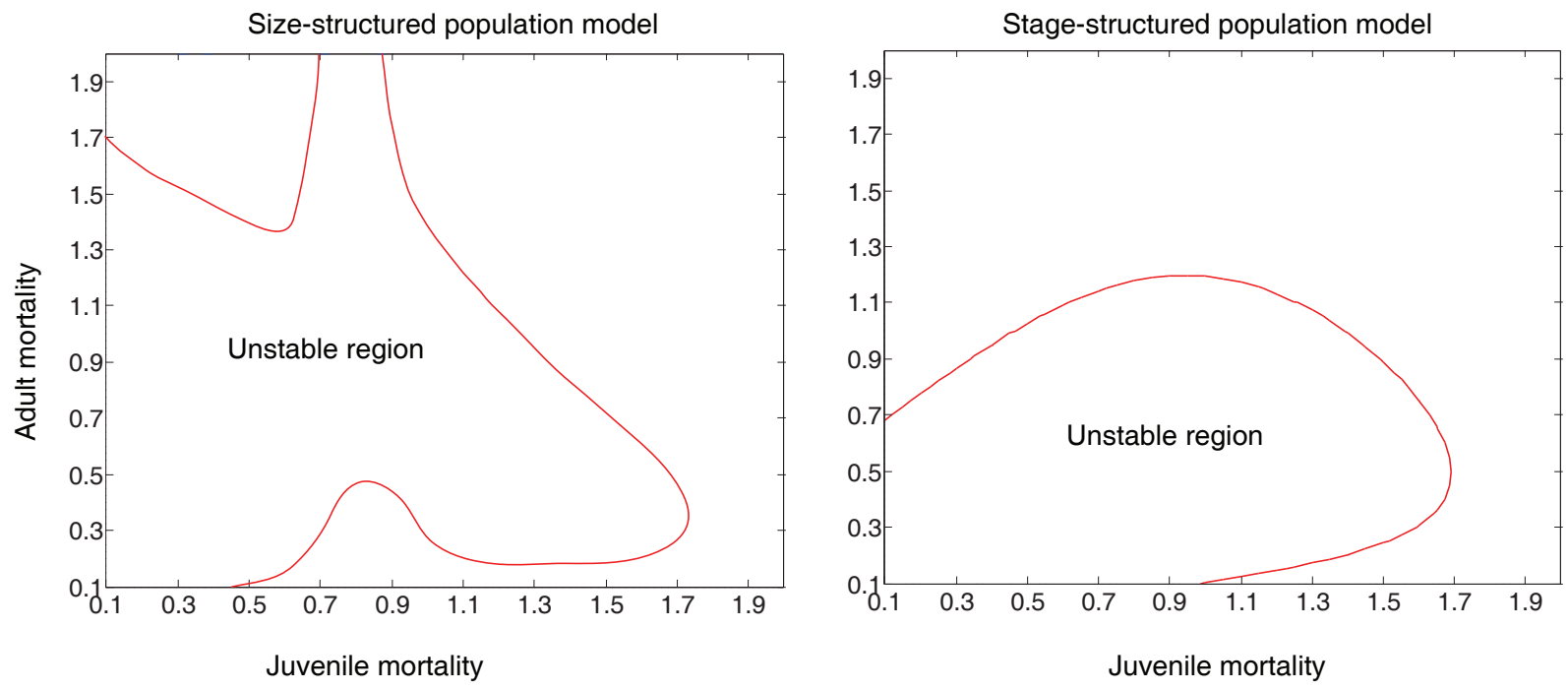

Fig. 7: Stability region of the size-structured population model (shaded area) and the stage-structured population model (hatched area) in the parameter space spanned by the two bifurcation parameters of juvenile $\left(\mu_{j}\right)$ and adult $\left(\mu_{a}\right)$ mortality for the case that adults are competitively stronger than juvenile (i.e., $q=2$ ). Other parameter values are taken as the default values in Table 1.

Claessen D, Van Oss C, de Roos AM, Persson L (2002) The impact of size-dependent predation on population dynamics and individual life history. Am Nat 83:1660-1675.

Claessen D, de Roos AM (2003) Bistability in a size-structured population model of cannibalistic fish a continuation study. Theor Popul Biol 64:49-65.

Datta S, Delius GW, Law R (2010) A jump-growth model for predator-prey dynamics: derivation and application to marine ecosystems. Bull Math Biol 72:1361-1382.

de Roos AM, Metz JAJ, Evers E, Leipoldt A (1990) A size-dependent predator-prey interactions: who pursues whom? J Math Biol 28:609-643.

de Roos AM, Persson L, 2002. Size-dependent life-history traits promotes catastrophic collapses of top predators. Proc Natl Acad Sci USA 99:12907-12912.

de Roos AM, Persson L., 2003. Competition in size-structured populations: mechanisms inducing cohort formation and population cycles. Theor Popul Biol 63:1-16.

de Roos AM, Persson L, McCauley E (2003) The influence of size-dependent life-history traits on the structure and dynamics of populations and communities. Ecol Lett 6:473-487.

de Roos AM, Schellekens T, Van Kooten T, Van De Wolfshasr K, Claessen D, Persson L (2008) Simplifying a physiologically structured population model to a stage-structured biomass model. Theor Popul Biol $73: 47-62$.

de Roos AM, Diekmann O, Getto P, Kirkilionis MA (2010) Numerical equilibrium analysis for structured consumer resource models. Bull Math Biol 72:259-297.

de Roos AM, Metz JAJ, Persson L (2013) Ontogenetic symmetry and asymmetry in energetics. J Math Biol 66: 889-914.

Diekmann O, Getto P, Gyllenberg M (2007) Stability and bifurcation analysis of voltterra functional equations in the light of suns and stars. SIAM J Math Anal 39:1023-1069. 
Diekmann O, Gyllenberg M, Metz JAJ, Nakaoka S, de Roos AM (2010) Daphnia revisited: local stability and bifurcation theory for physiologically structured population models explained by way of an example. J Math Biol 61: 277-318.

Diekmann O, Metz JAJ (2010) How to life a model for individual behavior to the population level? Phil Trans R Soc B 365:3523-3530.

Hartvig M, Andersen KH, Beyer JE (2011) Food web framework for size-structured populations. J Theor Biol 272:113-122.

Metz JAJ, Diekmann O (1986) Dynamics of physiologically structured populations. Lecture notes in Biomathematics, vol 68, Springer, Heidelberg.

Persson L, Leonardsson K, de Roos AM, Gyllenberg M, Christensen B (1998) Ontogenetic scaling of foraging rates and the dynamics of a size-structured consumer-resource model, Theor Popul Biol 54:270293

Werner EE (1988) Size, scaling and the evolution of complex life cycles. In: Ebenman B Persson L (eds), Size-structured Populations: Ecology and Evolution. Springer, Heidelberg, pp:60-81.

Woodward G, Ebenman B, Ernmerson M, Montoya JM, Olesen JM, Valido A, Warren PH (2005) Body size in ecological networks. Trends Ecol Evol 20:402-409

Zhang L, Lin ZG, Pedersen M (2012) Effects of growth curve plasticity on size-structured population dynamics. Bull Math Biol 74:327-345.

Zhang L, Thygesen UH, Knudsen K, Andersen KH (2013) Trait diversity promotes stability of community dynamics. Theor Ecol 6:57-69.

\section{Appendix A. The real and imaginary parts of $\boldsymbol{k}_{j}$}

In this appendix we present the analytical form of the real and imaginary parts of the $k_{j}$ in equations (4.38). Consider a purely imaginary $\lambda=i \omega(\omega>0)$ and plug it into equations (4.45), (4.46), (4.51) and (4.52).

Firstly we have

$$
\begin{aligned}
K^{r}\left(\tau^{*}, \lambda\right) & =\frac{s_{m} w_{j}^{\prime}\left(R^{*}\right)}{w_{j}^{2}\left(R^{*}\right)+\omega^{2}}\left[w_{j}\left(R^{*}\right)\left(\frac{s_{m}}{s_{0}} \cos \left(\omega \tau^{*}\right)-1\right)-\frac{s_{m}}{s_{0}} \omega \sin \left(\omega \tau^{*}\right)\right] \\
K^{i}\left(\tau^{*}, \lambda\right) & =-\frac{s_{m} w_{j}^{\prime}\left(R^{*}\right)}{w_{j}^{2}\left(R^{*}\right)+\omega^{2}}\left[w_{j}\left(R^{*}\right) \frac{s_{m}}{s_{0}} \sin \left(\omega \tau^{*}\right)+\omega\left(\frac{s_{m}}{s_{0}} \cos \left(\omega \tau^{*}\right)-1\right)\right] .
\end{aligned}
$$

For convenience, let

$$
I_{1}=\frac{\mu_{a}}{\mu_{a}+\lambda} e^{-\lambda \tau^{*}}
$$

and it is easy to see that

$$
\begin{aligned}
I_{1}^{r} & =\frac{\mu_{a}}{\mu_{a}^{2}+\omega^{2}}\left(b \cos \left(\omega \tau^{*}\right)-\omega \sin \left(\omega \tau^{*}\right)\right), \\
I_{1}^{i} & =-\frac{\mu_{a}}{\mu_{a}^{2}+\omega^{2}}\left(b \sin \left(\omega \tau^{*}\right)+\omega \cos \left(\omega \tau^{*}\right)\right) .
\end{aligned}
$$


Similarly, let

$$
I_{2}=\frac{1}{w_{j}\left(R^{*}\right)-\mu_{j}-\lambda}\left(\frac{\mu_{a}}{w_{a}\left(R^{*}\right)} e^{-\lambda \tau^{*}}-1\right)
$$

and

$$
\begin{aligned}
I_{2}^{r} & =\frac{1}{\left(w_{j}\left(R^{*}\right)-\mu_{j}\right)^{2}+\omega^{2}}\left[\left(w_{j}\left(R^{*}\right)-\mu_{j}\right)\left(\frac{\mu_{a}}{w_{a}\left(R^{*}\right)} \cos \left(\omega \tau^{*}\right)-1\right)+\omega \sin \left(\omega \tau^{*}\right) \frac{\mu_{a}}{w_{a}\left(R^{*}\right)}\right] \\
I_{2}^{i} & =\frac{1}{\left(w_{j}\left(R^{*}\right)-\mu_{j}\right)^{2}+\omega^{2}}\left[-\left(w_{j}\left(R^{*}\right)-\mu_{j}\right) \frac{\mu_{a}}{w_{a}\left(R^{*}\right)} \sin \left(\omega \tau^{*}\right)+\omega\left(\frac{\mu_{a}}{w_{a}\left(R^{*}\right)} \cos \left(\omega \tau^{*}\right)-1\right)\right]
\end{aligned}
$$

Thus, we have $k_{1}^{r}=I_{1}^{r}$ and $k_{1}^{i}=I_{1}^{i}$. Moreover,

$$
\begin{aligned}
k_{2}^{r}= & \frac{w_{a}^{\prime}\left(R^{*}\right)}{w_{a}\left(R^{*}\right)}+\frac{\mu_{j}-\mu_{a}}{s_{m} w_{j}\left(R^{*}\right)}\left(K^{r}\left(\tau^{*}, \lambda\right) I_{1}^{r}-K^{i}\left(\tau^{*}, \lambda\right) I_{1}^{i}\right) \\
& +\frac{w_{a}\left(R^{*}\right)}{s_{0} w_{j}\left(R^{*}\right)} F\left(\tau^{*}\right)\left(K^{r}\left(\tau^{*}, \lambda\right) \cos \left(\omega \tau^{*}\right)+K^{i}\left(\tau^{*}, \lambda\right) \sin \left(\omega \tau^{*}\right)\right), \\
k_{2}^{i}= & \frac{\mu_{j}-\mu_{a}}{s_{m} w_{j}\left(R^{*}\right)}\left(K^{r}\left(\tau^{*}, \lambda\right) I_{1}^{i}+K^{i}\left(\tau^{*}, \lambda\right) I_{1}^{r}\right) \\
& +\frac{w_{a}\left(R^{*}\right)}{s_{0} w_{j}\left(R^{*}\right)} F\left(\tau^{*}\right)\left(-K^{r}\left(\tau^{*}, \lambda\right) \sin \left(\omega \tau^{*}\right)+K^{i}\left(\tau^{*}, \lambda\right) \cos \left(\omega \tau^{*}\right)\right),
\end{aligned}
$$

and

$$
\begin{aligned}
k_{3}^{r} & =-\frac{s_{0} I R^{*}}{H+R^{*}} I_{2}^{r}-q s_{0} \frac{I R^{*}}{H+R^{*}} \frac{1}{w_{a}\left(R^{*}\right)} I_{1}^{r}, \\
k_{3}^{i} & =-\frac{s_{0} I R^{*}}{H+R^{*}} I_{2}^{i}-q s_{0} \frac{I R^{*}}{H+R^{*}} \frac{1}{w_{a}\left(R^{*}\right)} I_{1}^{i}
\end{aligned}
$$

Finally,

$$
\begin{aligned}
k_{4}^{r}= & -\frac{I H}{\left(H+R^{*}\right)^{2}}\left[\frac{s_{0}}{w_{j}\left(R^{*}\right)-\mu_{j}}\left(\frac{\mu_{a}}{w_{a}\left(R^{*}\right)}-1\right)+\frac{q s_{0}}{w_{a}\left(R^{*}\right)}\right] \\
& \left.-\frac{\mu_{j}-\mu_{a}}{s_{m} w_{j}\left(R^{*}\right)} \frac{I R^{*}}{H+R^{*}} \frac{q s_{0}}{w_{a}\left(R^{*}\right)}\left(K^{r}\left(\tau^{*}, \lambda\right) I_{1}^{r}-K^{i}\left(\tau^{*}, \lambda\right) I_{1}^{i}\right)\right) \\
& -\frac{(1-q)}{s_{m} w_{j}\left(R^{*}\right)} \frac{I R^{*}}{H+R^{*}} F\left(\tau^{*}\right)\left(K^{r}\left(\tau^{*}, \lambda\right) \cos \left(\omega \tau^{*}\right)+K^{i}\left(\tau^{*}, \lambda\right) \sin \left(\omega \tau^{*}\right)\right) \\
& -\frac{I R^{*}}{H+R^{*}} \frac{s_{0} w_{j}^{\prime}\left(R^{*}\right)}{w_{j}^{2}\left(R^{*}\right)+\omega^{2}}\left[w_{j}\left(R^{*}\right)\left(\frac{1}{2 w_{j}\left(R^{*}\right)-\mu_{j}}\left(\frac{s_{m} \mu_{a}}{s_{0} w_{a}\left(R^{*}\right)}-1\right)-I_{2}^{r}\right)-\omega I_{2}^{i}\right], \\
k_{4}^{i}= & \left.-\frac{\mu_{j}-\mu_{a}}{s_{m} w_{j}\left(R^{*}\right)} \frac{I R^{*}}{H+R^{*}} \frac{q s_{0}}{w_{a}\left(R^{*}\right)}\left(K^{r}\left(\tau^{*}, \lambda\right) I_{1}^{i}+K^{i}\left(\tau^{*}, \lambda\right) I_{1}^{r}\right)\right) \\
& -\frac{(1-q)}{s_{m} w_{j}\left(R^{*}\right)} \frac{I R^{*}}{H+R^{*}} F\left(\tau^{*}\right)\left(-K^{r}\left(\tau^{*}, \lambda\right) \sin \left(\omega \tau^{*}\right)+K^{i}\left(\tau^{*}, \lambda\right) \cos \left(\omega \tau^{*}\right)\right) \\
& -\frac{I R^{*}}{H+R^{*}} \frac{s_{0} w_{j}^{\prime}\left(R^{*}\right)}{w_{j}^{2}\left(R^{*}\right)+\omega^{2}}\left[-w_{j}\left(R^{*}\right) I_{2}^{i}-\omega\left(\frac{1}{2 w_{j}\left(R^{*}\right)-\mu_{j}}\left(\frac{s_{m} \mu_{a}}{s_{0} w_{a}\left(R^{*}\right)}-1\right)-I_{2}^{r}\right)\right] .
\end{aligned}
$$

\section{Appendix B. Stability analysis of the stage-structured population model}

In this appendix we present the method by which the stability of the equilibrium $\left(J^{*}, A^{*}, R^{*}\right)$ in the stage-structured population model (2.8) can be determined. 
The stability of the positive equilibrium $\left(J^{*}, A^{*}, R^{*}\right)$ is governed by the following characteristic equation, which is easy to obtain after linearising the stage-structured population model around the equilibrium,

$$
\left|\begin{array}{ccc}
a_{11}-\lambda & a_{12} & a_{13} \\
a_{21} & a_{22}-\lambda & a_{23} \\
a_{31} & a_{32} & a_{33}-\lambda
\end{array}\right|=0
$$

where

$$
\begin{aligned}
& a_{11}=-w_{a}\left(R^{*}\right) A^{*} / J^{*}, \quad a_{12}=w_{a}\left(R^{*}\right), \quad a_{13}=\frac{\partial w_{a}\left(R^{*}\right)}{\partial R} A^{*}+\frac{\partial w_{j}\left(R^{*}\right)}{\partial R} J^{*}-\frac{\partial \gamma\left(w_{j}\left(R^{*}\right)\right)}{\partial R} J^{*}, \\
& a_{21}=\gamma\left(w_{j}\left(R^{*}\right)\right), \quad a_{22}=-\mu_{a}, \quad a_{23}=\frac{\partial \gamma\left(w_{j}\left(R^{*}\right)\right)}{\partial R} J^{*}, \\
& a_{31}=-I \frac{R^{*}}{H+R^{*}}, \quad a_{32}=-q I \frac{R^{*}}{H+R^{*}}, \quad a_{33}=G^{\prime}\left(R^{*}\right)-\frac{H I\left(J^{*}+q A^{*}\right)}{\left(H+R^{*}\right)^{2}} .
\end{aligned}
$$

Alternatively, there is

$$
\lambda^{3}+a_{1} \lambda^{2}+a_{2} \lambda+a_{3}=0
$$

where

$$
\begin{aligned}
& a_{1}=-\left(a_{11}+a_{22}+a_{33}\right), \\
& a_{2}=a_{22} a_{33}-a_{23} a_{32}+a_{11} a_{33}+a_{13} a_{31}, \\
& a_{3}=a_{11} a_{23} a_{32}+a_{13} a_{31} a_{22}-a_{12} a_{23} a_{31}-a_{13} a_{21} a_{32} .
\end{aligned}
$$

Applying the Routh-Hurwitz criterion, we can easily determine the local stability of the equilibrium. 